\title{
Vitreous metabolomics profiling of proliferative diabetic retinopathy
}

\author{
Yohei Tomita $^{1,2}$ (1) $\cdot$ Gael Cagnone $^{3} \cdot$ Zhongjie Fu $^{1,4} \cdot$ Bertan Cakir $^{1} \cdot$ Yumi Kotoda $^{1} \cdot$ Masaki Asakage $^{5} \cdot$ \\ Yoshihiro Wakabayashi ${ }^{5}$. Ann Hellström ${ }^{6}$. Jean-Sébastien Joyal ${ }^{3,7}$. Saswata Talukdar ${ }^{8}$. Lois E. H. Smith ${ }^{1}$ (1) \\ Yoshihiko Usui ${ }^{5}$
}

Received: 2 July 2020 / Accepted: 10 September 2020 / Published online: 25 October 2020

(C) Springer-Verlag GmbH Germany, part of Springer Nature 2020

\begin{abstract}
Aims/hypothesis Proliferative diabetic retinopathy (PDR) with retinal neovascularisation (NV) is a leading cause of vision loss. This study identified a set of metabolites that were altered in the vitreous humour of PDR patients compared with non-diabetic control participants. We corroborated changes in vitreous metabolites identified in prior studies and identified novel dysregulated metabolites that may lead to treatment strategies for PDR.

Methods We analysed metabolites in vitreous samples from 43 PDR patients and 21 non-diabetic epiretinal membrane control patients from Japan (age 27-80 years) via ultra-high-performance liquid chromatography-mass spectrometry. We then investigated the association of a novel metabolite (creatine) with retinal NV in mouse oxygen-induced retinopathy (OIR). Creatine or vehicle was administered from postnatal day (P)12 to P16 (during induced NV) via oral gavage. P17 retinas were quantified for NV and vaso-obliteration.

Results We identified 158 metabolites in vitreous samples that were altered in PDR patients vs control participants. We corroborated increases in pyruvate, lactate, proline and allantoin in PDR, which were identified in prior studies. We also found changes in metabolites not previously identified, including creatine. In human vitreous humour, creatine levels were decreased in PDR patients compared with epiretinal membrane control participants (false-discovery rate $<0.001$ ). We validated that lower creatine levels were associated with vascular proliferation in mouse retina in the OIR model $(p=0.027)$ using retinal metabolomics. Oral creatine supplementation reduced NV compared with vehicle (P12 to P16) in OIR $(p=0.0024)$.

Conclusions/interpretation These results suggest that metabolites from vitreous humour may reflect changes in metabolism that can be used to find pathways influencing retinopathy. Creatine supplementation could be useful to suppress NV in PDR.
\end{abstract}

Keywords Creatine - Metabolomics · Oxygen-induced retinopathy · Proliferative diabetic retinopathy · Retinal neovascularisation

Yohei Tomita and Gael Cagnone contributed equally to this work.

Electronic supplementary material The online version of this article (https://doi.org/10.1007/s00125-020-05309-y) contains peer-reviewed but unedited supplementary material, which is available to authorised users.

Yoshihiko Usui usuyoshi@gmail.com

$\triangle$ Lois E. H. Smith

Lois.Smith@childrens.harvard.edu

1 Department of Ophthalmology, Boston Children's Hospital, Harvard Medical School, Boston, MA, USA

2 Department of Ophthalmology, Keio University School of Medicine, Tokyo, Japan

3 Department of Pediatrics, Pharmacology and Ophthalmology, CHU Sainte-Justine Research Center, Université de Montréal, Montreal, QC, Canada
4 Manton Center for Orphan Disease, Boston Children's Hospital, Harvard Medical School, Boston, MA, USA

5 Department of Ophthalmology, Tokyo Medical University Hospital, Tokyo, Japan

6 Pediatric Ophthalmology, Institute of Neuroscience and Physiology, Sahlgrenska Academy, Gothenburg University,

Gothenburg, Sweden

7 Department of Pharmacology and Therapeutics, McGill University, Montreal, QC, Canada

8 Merck \& Co., Inc., South San Francisco, CA, USA 


\section{Research in context}

\section{What is already known about this subject?}

- Diabetic retinopathy is a leading cause of vision loss worldwide; current treatments do not always suppress retinal neovascularisation

- Vitreous metabolites may reflect novel pathways involved in neovascularisation

- A few studies have shown that some vitreous metabolite levels are increased or decreased in diabetic retinopathy compared with non-diabetic control participants, but none have suggested new potential pathways that might lead to new treatment options

\section{What is the key question?}

- Does our data analysis validate previously published studies of vitreous metabolites in proliferative diabetic retinopathy (PDR) and identify new metabolites and pathways that may lead to novel treatment strategies?

\section{What are the new findings?}

- We validated many vitreous metabolites found in diabetic retinopathy in other studies; we also found a novel target: creatine levels were decreasedin vitreous humourof PDR patients comparedwith control participants

- We found that creatine levels were also decreased in the mouse oxygen-induced retinopathy model vs normoxia

- Creatine supplementation suppressed retinal neovascularisation in oxygen-induced retinopathy in mice

\section{How might this impact on clinical practice in the foreseeable future?}

- We documented that creatine supplementation inhibited pathological neovascularisation in a mouse model of proliferative retinopathy, suggesting that creatine metabolism is a potential target in PDR

$\begin{array}{ll}\text { Abbreviations } \\ \text { ERM } & \text { Epiretinal membrane } \\ \text { FDR } & \text { False-discovery rate } \\ \text { NV } & \text { Neovascularisation } \\ \text { OIR } & \text { Oxygen-induced retinopathy } \\ \text { P } & \text { Postnatal day } \\ \text { PCA } & \text { Principal component analysis } \\ \text { PDGF } & \text { Platelet-derived growth factor } \\ \text { PDGFR } & \text { Platelet-derived growth factor receptor } \\ \text { PDR } & \text { Proliferative diabetic retinopathy } \\ \text { VEGF } & \text { Vascular endothelial growth factor } \\ \text { VO } & \text { Vaso-obliteration }\end{array}$

\section{Introduction}

The prevalence of diabetes is increasing and diabetic retinopathy is a leading cause of vision loss worldwide [1]. Proliferative diabetic retinopathy (PDR), which is the most advanced stage of diabetic retinopathy, is characterised by neovascularisation (NV) and is associated with macular oedema, tractional retinal detachment and haemorrhage, which lead to vision loss [2]. The current therapeutic strategies for PDR, such as laser photocoagulation and anti-vascular endothelial growth factor (VEGF) injections, do not always control disease progression. Moreover, anti-VEGF treatment usually needs multiple applications and is challenging in patients with recent thromboembolic events or myocardial infarction [3], and may also result in hypertension and increased cardiovascular events [4]. Thus, it is important to identify new pathways underlying retinal $\mathrm{NV}$ in order to develop alternative preventative therapeutic approaches to treat PDR. We hypothesised that metabolite profiling of vitreous humour from patients with PDR vs patients without retinal NV (non-diabetes mellitus) may reveal new targetable pathways underlying pathological blood vessel growth.

Metabolomics, which is based on the principles of genomics, transcriptomics and proteomics, aims to identify metabolites with low molecular mass (<1500 Da) such as lipids, amino acids, nucleotides, vitamins and polyols. By-products of tissue metabolism, metabolites can reveal intrinsic changes in tissue homeostasis as well as the extrinsic effects of exogenous factors such as diet, medications and host gut microbiome [5].

In ophthalmology, composition of the vitreous humour has been used as a read-out of retinal metabolism [6, 7]. In PDR patients, biochemical alterations in the vitreous humour closely reflect changes in retinal homeostasis [8]. Barba et al demonstrated that lactate is increased while ascorbic acid is decreased [9]; Paris et al reported that arginine, proline and allantoin are increased [10]; and Haines et al reported that pyruvate and purine-related pathways are increased [6]. 
Here, an untargeted metabolomics study was performed to reveal the metabolomic profiles of the vitreous humour of patients with PDR compared with non-diabetic control participants.

\section{Methods}

Study ethics approval The clinical study was part of a crosssectional observational study of vitreous metabolites at Tokyo Medical University. The research protocol was conducted in accordance with Helsinki protocols and was approved by the Institutional Review Board of Tokyo Medical University (number 2311). Patient data and vitreous samples were handled according to the Declaration of Helsinki. Written informed consent for the surgery, and for collection of the clinical data and vitreous samples, was provided by all patients. All animal studies were approved by the Institutional Animal Care and Use Committee at Boston Children's Hospital (Animal Resources at Children's Hospital, protocol number 19-04-3913R) and adhered to the Association for Research in Vision and Ophthalmology Statement.

Collection of human vitreous samples Vitreous samples $(0.5-$ $1 \mathrm{ml}$ ) were collected at the time of medically indicated surgery, from 43 patients with PDR and 21 non-diabetic control patients with epiretinal membrane (ERM, nondiabetes mellitus) undergoing membrane removal (age 2780 years). The criteria for the surgery for PDR were vitreous haemorrhage dense enough to obscure any view of the fundus (three or $7.0 \%$ ), tractional retinal detachment (ten or 23.3\%), macular oedema (ten or $23.3 \%$ ) and fibrovascular membrane (22 or $51.2 \%$ ). Under local anaesthesia, patients underwent standard pars plana vitrectomy as described [10]. Briefly, at the beginning of the surgery, vitreous samples were aspirated and then stored at $-80^{\circ} \mathrm{C}$. Patients with cataracts were treated with phacoemulsification and aspiration. Then, vitrectomy was conducted with a vitreous cutter (5000 cycles/min).

Human vitreous samples preparation for ultra-highperformance LC-MS/MS Vitreous samples were extracted and split into five equal parts for metabolomics analysis on the ultra-high-performance LC-MS/MS and Polar LC platforms (Metabolon, Morrisville, NC, USA). As described by Bailey et al [11], metabolomics measurements of the extracted and dried samples were performed using a Waters ACQUITY UPLC (Waters Corporation, Milford, MA, USA). Then, measurements were performed with a Thermo Scientific QExactive high-resolution/accurate mass spectrometer interfaced with a heated electrospray ionisation (HESI-II) source, and an Orbitrap mass analyser operated at 35,000 mass resolution (Thermo Fisher Scientific, Waltham, MA, USA).
Metabolomics data processing and statistical analysis for human vitreous humour Using Metabolon proprietary informatic software (mLims 6.0.1, Metabolon, Morrisville, NC, USA), the ions were matched to an in-house library for metabolite identification and metabolite quantification by peak area integration.

Briefly, raw data are extracted, peak identified and quality control processed using Metabolon's hardware and software (mLims 6.0.1, Metabolon). Compounds are identified by comparison with purified standards or unknown recurrence entities as described by Serrano et al [12].

Raw metabolomics data from human vitreous samples identified by Metabolon were analysed by MetaboAnalyst $4.0[13$, 14]. Briefly, metabolomic datasets from each patient were filtered (interquantile range), normalised (quantile), log transformed and scaled across samples. MetaboAnalyst random forest classifier identified outliers that could be traced back to patients suffering from uveitis, which led to the removal of all uveitis-associated samples from further analysis. Principal component analysis (PCA) was performed using the prcomp package within MetaboAnalyst software in order to find in an unsupervised manner the components that best explain the variance in the dataset. Hierarchical clustering was performed with the hclust function in the stat package using Euclidean distance and the Ward clustering algorithm; the result was visualised as a heatmap. Pathway analysis was performed using metabolite enrichment set analysis (MESA) or metabolite set enrichment analysis [15] in order to identify biologically meaningful patterns in metabolite concentration changes between sample groups. Differences in metabolite levels were assessed using unpaired $t$ test with false-discovery rate (FDR) adjustment and significance less than 0.05 and were visualised by volcano plot.

\section{Collection of mouse retina samples following oxygen-induced} retinopathy The mouse oxygen-induced retinopathy (OIR) model was used as described [16-18]. Briefly, to induce retinal NV, mouse pups (C57BL/6J, male and female; Jackson laboratory, Bar Harbor, ME, USA) and their nursing mother were exposed to $75 \pm 3 \%$ oxygen from postnatal day $(\mathrm{P}) 7$ to $\mathrm{P} 12$ and then returned to room air until P17. All mice were housed with a $12 \mathrm{~h} \mathrm{light/dark} \mathrm{cycle.} \mathrm{At} \mathrm{P17,} \mathrm{mice} \mathrm{were}$ euthanised by ketamine hydrochloride and xylazine hydrochloride, and retinas were dissected and frozen immediately in liquid nitrogen and stored at $-80^{\circ} \mathrm{C}$. Six retinas were pooled as one replicate to reduce biological variability with metabolomics analysis, and 3-4 independent replicates of six pooled retinas per group were assessed for OIR and control normoxic conditions.

Preparation of mouse retina samples for MS-based metabolomics and hybrid metabolomics data processing and analysis Samples were analysed with LC-MS/MS by the NYU Metabolomics Core Resource Laboratory, New York, NY, 
USA. Briefly, samples were subjected to an LC-MS analysis to detect and quantify known peaks. Metabolites were extracted from each sample, as described [19], scaling the metabolite extraction volume to the tissue mass for each sample (10 mg/ $\mathrm{ml})$. Then, LC-MS analyses were carried out and all data were acquired. Relative quantification of metabolites was performed [20,21, 22], and metabolomics informatics were performed as described [23]. Student's $t$ tests were performed with the Python SciPy (1.1.0, SciPy.org, https://pypi.org/ project/scipy/1.1.0/) library to test for differences and $p$ value $<0.05$ was considered significant. Heatmaps were generated with hierarchical clustering performed on the imputed matrix values utilising the R library heatmap (1.0. 12, https://rdrr.io/cran/pheatmap/).

Quantification of pathological angiogenesis in retinas following creatine treatment In OIR, mouse pups (C57BL/6J, male and female) were orally gavaged with creatine monohydrate (500 mg/kg; C3630; Sigma-Aldrich, St Louis, MO, USA) or PBS (control; 10010-023; Thermo Fisher Scientific) daily, from P12 to P16. Five mice each were allocated to the creatine treatment group and to the vehicle group. At P17, eyes were enucleated and retinas were stained with Alexa Fluor 594conjugated isolectin GS-IB4 $(10 \mu \mathrm{g} / \mathrm{ml}$; I21413; Thermo Fisher Scientific), as described [24]. Then, retinas were visualised using an epifluorescence microscope (AxioObserver.Z1; Carl Zeiss Microscopy, Jena, Germany). Mice weighing less than $5.0 \mathrm{~g}$ were excluded from this study [25]. At P17, the areas of retinal NV and vaso-obliteration (VO) were quantified as previously described [26].

Quantitative real-time RT-PCR Quantitative real-time PCR was performed as previously described [27]. Briefly, retina was isolated and homogenised with QIAzol Lysis Reagent (79306; Qiagen, Germantown, MD, USA). Then, supernatant was extracted using $20 \%$ chloroform and collected for RNA extraction with a PureLink RNA Mini Kit (12183025; Invitrogen, Grand Island, NY, USA). Next, RNA was reverse transcribed with iScript Reverse Transcription Supermix for RT-qPCR (1708841; BioRad, Hercules, CA, USA) using an S1000 Thermal cycler (1852148; BioRad). mRNA was quantified with an Applied Biosystems 7300 Real-Time PCR system software (Thermo Fisher Scientific) using 2xSYBR Green qPCR Master mix (B21202; bimake.com, Houston, TX, USA). Gene expression was calculated relative to Cyclophilin- $A$ internal control with the $\Delta \Delta C_{t}$ method. The primers are listed in electronic supplementary material (ESM) Table 1.

Statistical analysis for preclinical data Animal data are presented as mean \pm SEM. Two-tailed unpaired Student's $t$ test was used as specified in the figure legends (Prism v8.0; GraphPad Software, San Diego, CA, USA). Statistically significant difference was set at $p<0.05$.

\section{Results}

Clinical data of PDR and ERM patients Patients underwent surgery at the Ophthalmology Department of Tokyo Medical University between May 2015 and May 2017. Ten patients (two from control, eight from PDR groups) were excluded from the study because of any of following conditions: anti-VEGF intravitreal injection before vitrectomy, vitreous haemorrhage dense enough to obscure any view of the fundus, neovascular glaucoma or uveitis, in order to assess as homogeneous a group as possible. Thus, 19 non-diabetic control participants with ERM and 35 diabetic patients with PDR (two type 1 and 33 type 2) participated. Meta-data for patients are shown in Table 1 . The number of phakic patients was $16(84.2 \%)$ in the control group and $31(88.6 \%)$ in the PDR group. The number of prior intraocular surgeries was three $(15.8 \%)$ in control participants and four $(11.4 \%)$ in PDR. They included cataract surgery (six) in both groups and vitrectomy plus cataract surgery (one) in the control group. In the PDR group, no patients received intravitreal injection of triamcinolone. In the PDR group, 16 patients had nephropathy $(45.7 \%)$, nine patients had neuropathy $(25.7 \%)$ and five patients had heart disease $(14.3 \%)$. Ten patients $(28.6 \%)$ had macular oedema before vitrectomy. In addition, 13 PDR patients (37.1\%) were treated with insulin and 12 PDR patients $(34.3 \%)$ were treated with metformin. Nine PDR patients $(25.7 \%)$ had received photocoagulation.

Impact of PDR on vitreous metabolic profile Figure 1a shows a schema of the difference between patients with PDR and non-diabetes mellitus (ERM) in terms of retinal anatomy. To determine whether PDR patients could be discriminated from control patients (ERM) based on vitreous metabolite profiles, we performed PCA on metabolomic profiles of PDR and ERM vitreous samples, which showed a clear separation of PDR and ERM patients (PC1, 16.2\% of the variance) (Fig. 1b). The second component (PC2, explaining $12.3 \%$ of the variance) was associated with intra-variability within the PDR population. Using an FDR of 0.05 , we found 158 metabolites (out of 354 detected in vitreous samples) that were differentially present between the vitreous humour of PDR and ERM patients (Fig. 1c). Out of these 158 metabolites, 74 were increased in PDR samples while 84 were increased in ERM samples. As shown in Fig. 2, the top 30 metabolites with the highest statistical difference between PDR (class 1, blue) and control (class 2, yellow, ERM) patients were strongly discriminative of the two conditions. For example, allantoin, lactate, proline, dimethylglycine, $\alpha$-ketoglutarate and pyruvate were increased in the vitreous humour of PDR patients compared with ERM patients. To better understand the metabolic pathways associated with changes in the vitreous metabolic profiles of PDR patients, we performed metabolite set enrichment analysis of the top 50 most-altered metabolites (Fig. 3) 
Table 1 Clinical characteristics of the study population

\begin{tabular}{llll}
\hline Characteristic & Non-diabetes mellitus (ERM) & Diabetes mellitus (PDR) & $p$ value \\
\hline Total number & 19 & 35 & \\
Age (years; \pm SD) & $69.4 \pm 7.0$ & $58.1 \pm 13.6$ & $p<0.01$ \\
Sex $($ female; $\%)$ & 57.9 & 22.9 & $p<0.05$ \\
BMI $\left(\mathrm{kg} / \mathrm{m}^{2} ; \pm \mathrm{SD}\right)$ & $22.4 \pm 3.0$ & $24.4 \pm 3.2$ & $p<0.05$ \\
Phakic $(\%)$ & 84.2 & 88.6 & $p=0.69$ \\
Intraocular surgeries $(\%)$ & 15.8 & 11.4 & $p=0.69$ \\
$\mathrm{HbA}_{1 \mathrm{c}}(\mathrm{mmol} / \mathrm{mol})$ & n.a. & $54.8 \pm 20.6$ & - \\
$\mathrm{HbA}_{1 \mathrm{c}}(\%)$ & n.a. & $7.4 \pm 1.5$ & - \\
Complication & & & - \\
$\quad$ Nephropathy $(\%)$ & n.a. & 45.7 & - \\
$\quad$ Neuropathy $(\%)$ & n.a. & 25.7 & - \\
$\quad$ Heart disease $(\%)$ & n.a. & 14.3 & - \\
$\quad$ DME $(\%)$ & n.a. & 28.6 & - \\
Insulin treatment $(\%)$ & n.a. & 37.1 & - \\
Metformin treatment $(\%)$ & n.a. & 34.3 & - \\
Photocoagulation $(\%)$ & n.a. & 25.7 & \\
\hline
\end{tabular}

Sex, $\chi^{2}$ test; phakic and intraocular surgeries, Mann-Whitney $U$ test; others, Student's $t$ test n.a., not applicable; DME, diabetic macular oedema

Metabolomics analysis in human vitreous humour. (a)

Simplified schematic of the eye depicting the retinal vasculature in PDR (left-hand side) and ERM (right-hand side). (b) PCA between PDR (1, blue) and ERM (2, yellow). (c) Volcano plot of differentially expressed metabolites between PDR and ERM control samples (FDR 0.05). FC, fold change vs control; $\mathrm{PC} 1$, principal component 1 ; PC2, principal component 2

a

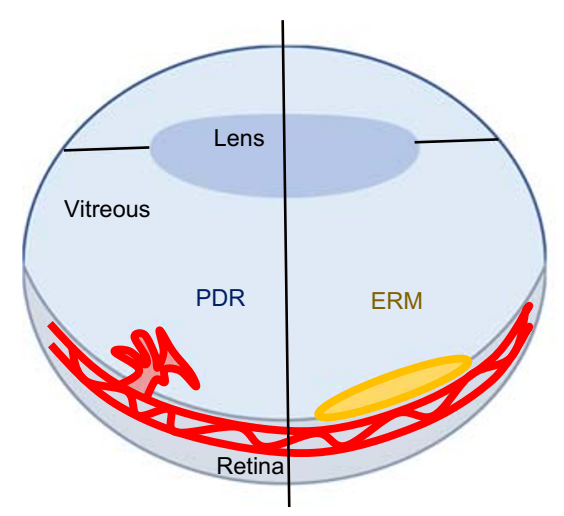

b

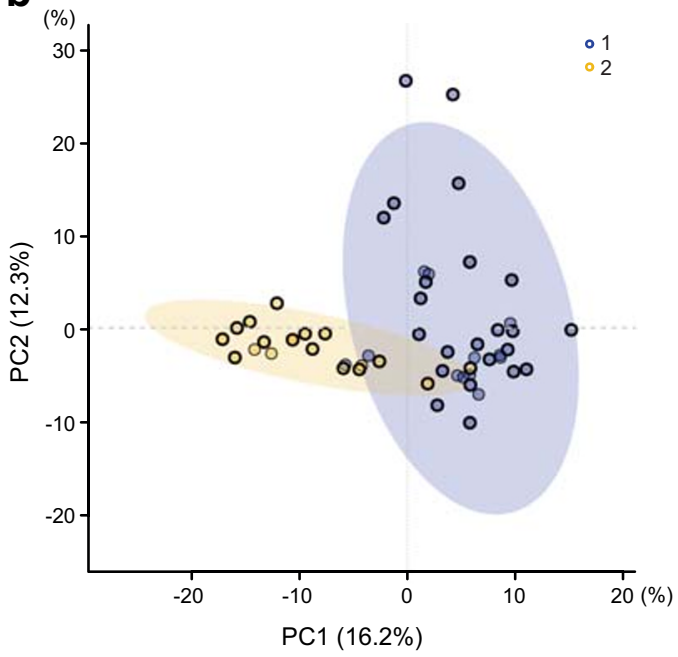

C

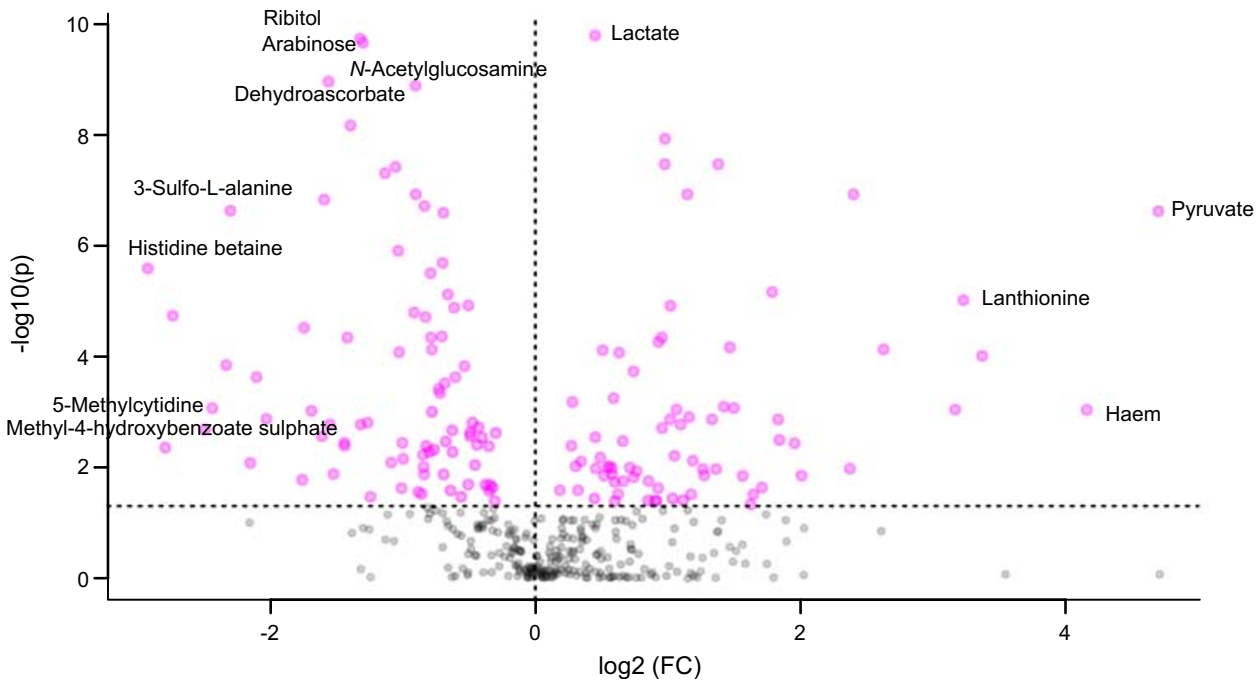


Fig. 2 Hierarchical clustering methods for metabolites in human vitreous humour. Heatmap of the top 30 differentially expressed metabolites. Columns represent the samples (control, PDR) and rows represent the metabolites. PDR (class 1, blue); ERM (class 2 , yellow). The relative levels of each metabolite are shown by the colours in the panel; the blue shows low levels, and the red shows high levels. The figure unit is normalised auto-scaled expression value

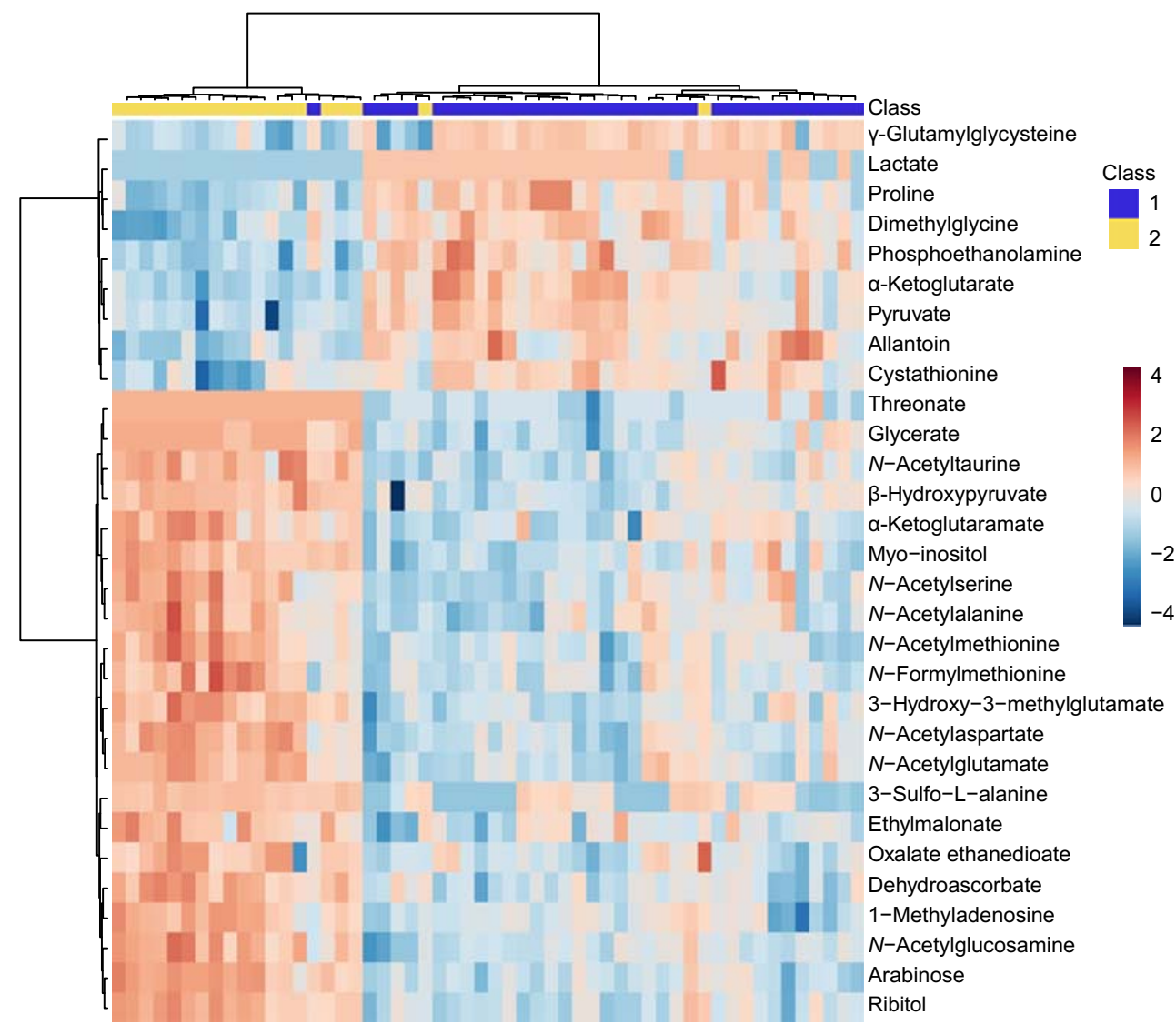

and found a significant enrichment in glycine, serine, arginine and proline amino acid metabolism.

Meta-data comparison with previous study To confirm the validity of the patient cohorts and our analysis, we compared our data with three similar published studies. Barba et al [9] analysed 22 type 1 diabetes patients with PDR and 22 patients with no diabetes with proton NMR ( ${ }^{1} \mathrm{H}$ NMR). They found that lactate was increased in vitreous humour of PDR patients compared with control participants. Paris et al [10] analysed 20 type 2 diabetes patients with PDR and 31 patients with no diabetes with HPLC-MS. They found that arginine, proline, allantoin, ornithine and other metabolites were increased in vitreous humour of PDR patients compared with control participants. Haines et al [6] analysed 17 type 1 and two type 2 diabetes patients with PDR compared with control participants. They found that pyruvate, inosine and other metabolites were increased in PDR vitreous samples compared with control samples. Our study also showed that lactate, pyruvate, proline, allantoin, urate, citrulline and ornithine were increased in PDR vitreous humour compared with control participants (Table 2, ESM Fig. 1). We did not see a correlation between PDR and the following metabolites that were reported previously: arginine, methionine, hypoxanthine and xanthine (Fig. 4a, ESM Fig. 2).
In contrast, inosine was modulated in the opposite direction in our study compared with previous publications (Table 2, ESM Fig. 2). We also found the following previously unreported metabolites that were significantly different between PDR vitreous and control vitreous samples: creatine, dimethyl glycine, $\mathrm{N}$ acetylserine, succinate and $\alpha$-ketoglutarate (Fig. 4b,c, ESM Fig. 2).

Glycine and creatine levels are associated with retinal NV Based on our metabolite set enrichment analysis, we found that creatine, a metabolite from the degradation of glycine, was present at lower levels in PDR vitreous samples (FDR $<0.001)$ while glycine was present at higher levels compared with ERM control samples (FDR <0.001) (Fig. 4b,c). Arginine, phosphocreatine and creatinine levels showed no significant differences between the two groups (Fig. 4a,d,e). To determine the validity of our findings with regard to the proliferative phenotype observed in diabetic retinopathy, we performed a metabolic analysis of mouse OIR retina. Figure 5a shows a schema of the difference between OIR and control normoxic retinas (normoxia) in terms of retinal anatomy. Samples were analysed with the hybrid LC-MS assay, and metabolites were quantified across the eight samples. A metabolomics heatmap of OIR is also shown as Fig. 5b. We found that creatine was decreased in OIR mouse retina. Similar to human PDR, we 
Fig. 3 Metabolite set enrichment analysis for human vitreous humour. Most-enriched metabolic pathways from the top 50 dysregulated metabolites in PDR samples compared with ERM control
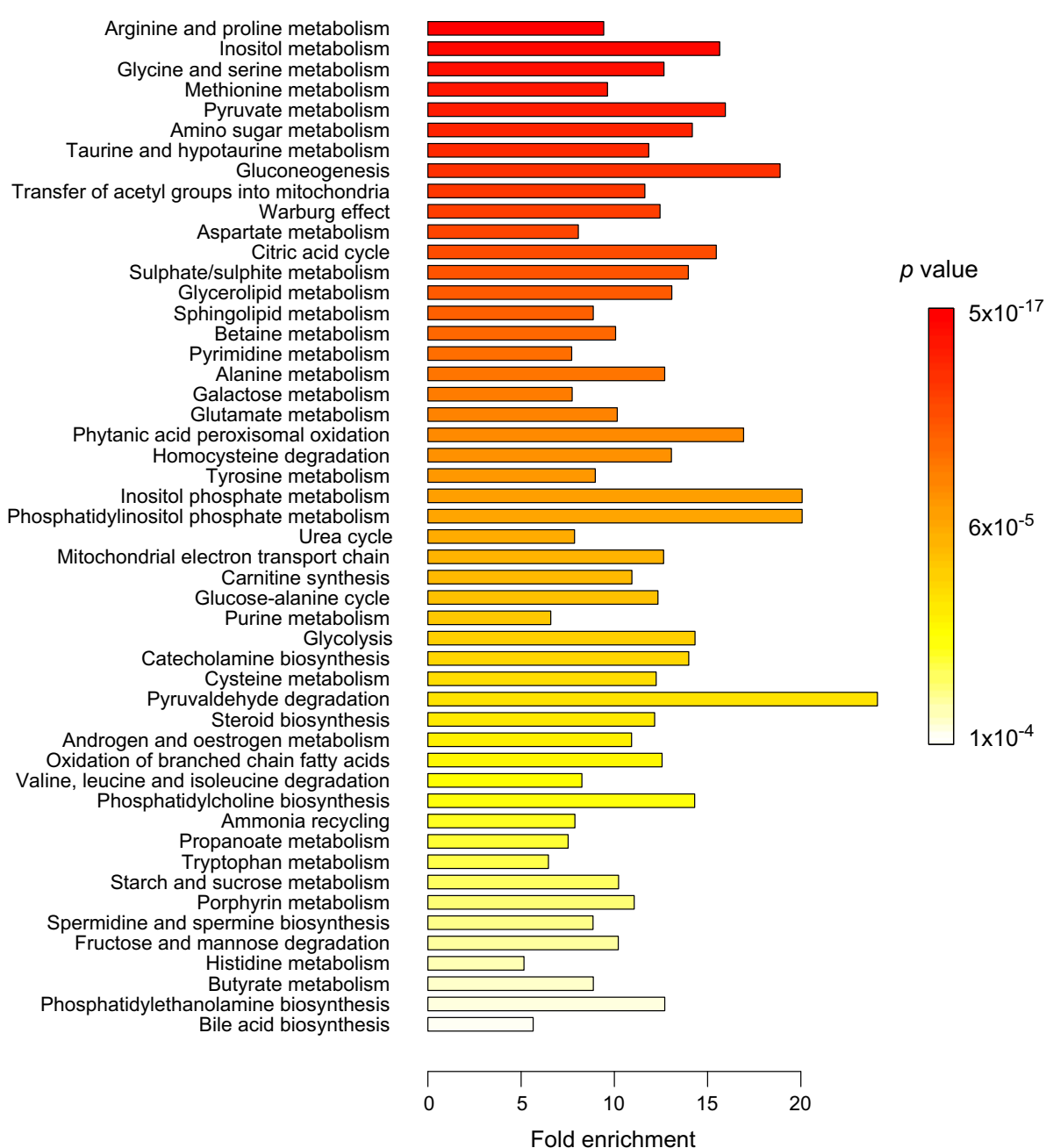

found a significantly lower level of creatine $(p=0.027, n=3,4)$ and a higher level of glycine $(p<0.001, n=3,4)$ in OIR retinas compared with control normoxic retinas in mice (Fig. 6a,b). Phosphocreatine and creatinine were not significantly different between the two groups (Fig. 6c,d). Figure 6e shows a schema of the glycine/creatine pathway in both PDR patients and the OIR model. Interestingly, pyruvate, proline, allantoin, citrulline and ornithine were increased significantly in OIR retinas compared with control normoxic retinas (ESM Fig. 3). These

Table 2 Summary of published studies on vitreous metabolomics of diabetic retinopathy

\begin{tabular}{|c|c|c|c|c|c|c|c|c|}
\hline Authors & Year & Samples & Platform & $\begin{array}{l}\text { Number } \\
\text { (cases) }\end{array}$ & $\begin{array}{l}\text { Number } \\
\text { (control) }\end{array}$ & Patients & Metabolites (up) & $\begin{array}{l}\text { Metabolites } \\
\text { (down) }\end{array}$ \\
\hline $\begin{array}{l}\text { Barba et al } \\
\quad[9]\end{array}$ & 2010 & $\begin{array}{l}\text { Vitreous } \\
\text { humour }\end{array}$ & ${ }^{1} \mathrm{H}$ NMR & $\begin{array}{l}22 \text { type } 1 \\
\text { diabetes } \\
\text { with PDR }\end{array}$ & $\begin{array}{l}22 \text { no diabetes } \\
(\mathrm{MH})\end{array}$ & & Lactate & $\begin{array}{l}\text { Galactitol, } \\
\text { ascorbic acid }\end{array}$ \\
\hline $\begin{array}{l}\text { Paris et al } \\
\quad[10]\end{array}$ & 2016 & $\begin{array}{l}\text { Vitreous } \\
\text { humour }\end{array}$ & HPLC-MS & $\begin{array}{l}20 \text { type } 2 \\
\text { diabetes } \\
\text { with PDR }\end{array}$ & $\begin{array}{l}31 \text { no diabetes } \\
(\text { ERM, MH) }\end{array}$ & Japanese & $\begin{array}{l}\text { Arginine, proline, allantoin, citrulline, } \\
\text { ornithine, octanoylcarnitine, } \\
\text { methionine, decanoylcarnitine }\end{array}$ & \\
\hline $\begin{array}{l}\text { Haines } \\
\text { et al [6] }\end{array}$ & 2018 & $\begin{array}{l}\text { Vitreous } \\
\text { humour }\end{array}$ & UHPLC-MS & $\begin{array}{l}17 \text { type } 1 \text { and } 2 \\
\text { diabetes } \\
\text { with PDR }\end{array}$ & $\begin{array}{l}18 \text { no diabetes } \\
(\text { ERM })\end{array}$ & $\begin{array}{l}\text { White, Asian, } \\
\text { African, } \\
\text { Hispanic }\end{array}$ & $\begin{array}{l}\text { Pyruvate, inosine, hypoxanthine, urate, } \\
\text { allantoate, pentose phosphates }\end{array}$ & Xanthine \\
\hline $\begin{array}{l}\text { Current } \\
\text { study }\end{array}$ & 2020 & $\begin{array}{l}\text { Vitreous } \\
\text { humour }\end{array}$ & UHPLC-MS & $\begin{array}{l}35 \text { type } 1 \text { and } 2 \\
\text { diabetes } \\
\text { with PDR }\end{array}$ & $\begin{array}{l}19 \text { no diabetes } \\
\text { (ERM) }\end{array}$ & Japanese & $\begin{array}{l}\text { Glycine, lactate, pyruvate, proline, } \\
\text { allantoin, urate, citrulline, ornithine, } \\
\text { dimethylglycine, } N \text {-acetylserine, } \\
\alpha \text {-ketoglutarate }\end{array}$ & $\begin{array}{l}\text { Creatine, } \\
\text { succinate }\end{array}$ \\
\hline
\end{tabular}

MH, macular hole; UHPLC-MS, ultra-high-performance liquid chromatography-mass spectrometry 
Fig. 4 The levels of the glycine/ creatine pathway in human PDR vitreous humour. $(\mathbf{a}, \mathbf{d}, \mathbf{e})$ No significant difference was detected for arginine,

phosphocreatine and creatinine.

(b) Creatine level was decreased in the vitreous humour of PDR patients compared with control participants. (c) Glycine level was increased in the vitreous humour of PDR patients compared with control participants. The data were assessed using unpaired $t$ test with FDR. ***FDR $<0.001$ $\mathrm{SEV}$, scaled expression value a

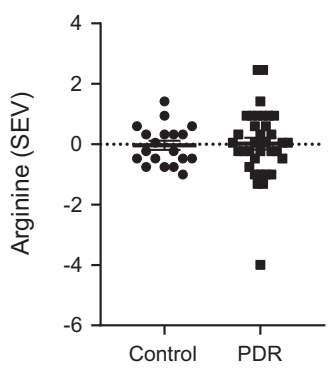

d

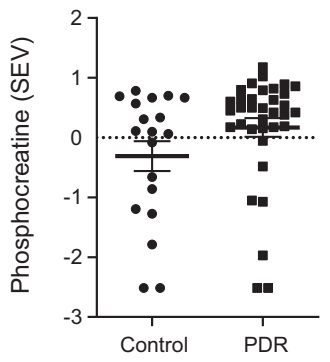

b

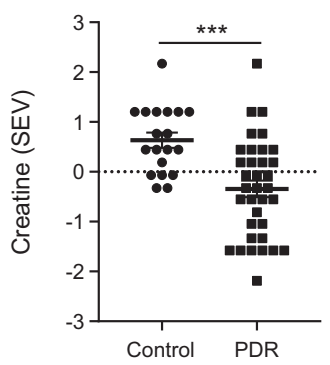

C

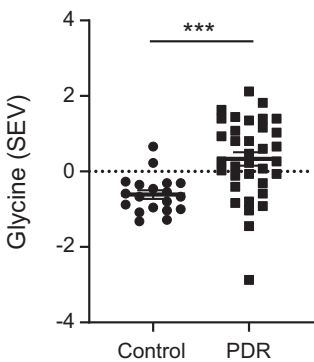

e

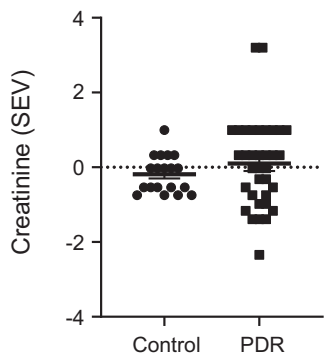

results are consistent with those of human PDR vitreous and control samples.

\section{Anti-angiogenic effect of oral administration of creatine on} the retina of OIR We used the OIR mouse model of retinal NV to investigate whether decreased creatine observed in proliferative retinopathy could contribute to the development of pathological retinal NV by exogenously increasing creatine in OIR. Figure 7a-d shows representative whole mounted retinas of creatine- and vehicle control-treated mice. In creatine-treated OIR, the NV area in the creatine group was significantly decreased compared with the vehicle group $(p=$ $0.0024, n=10$ ) (Fig. 7e). No significant differences in VO were detected among the groups $(n=10)$ (Fig. 7f). The body weight was comparable between the two groups (Fig. $7 \mathrm{~g}$ ), suggesting that the impact of creatine was not from systemic changes and that treatment was not toxic [28]. Furthermore, creatine treatment decreased the expression level of Vegf- $a$ and $P d g f-b$ mRNA in the OIR retina (ESM Fig. 4).

\section{Discussion}

Metabolomics analysis of vitreous samples from PDR patients is a potential tool to help identify new pathways associated with NV that may lead to novel treatments. In this study we identified 158 metabolites in vitreous humour that were significantly affected in PDR patients relative to non-diabetic control participants. We corroborated the associations of some metabolites with PDR that had been identified in prior studies, namely, an increase in pyruvate, lactate, proline, glycine, citrulline, ornithine and allantoin $[6,9,10,29]$. We also found novel metabolites associated with PDR including creatine, dimethylglycine, $\mathrm{N}$ acetylserine, succinate and $\alpha$-ketoglutarate. Also, compared with earlier publications, we did not observe metabolomic changes in arginine, methionine, hypoxanthine and xanthine associated with PDR.

Our study has limitations. A larger cohort of PDR patients might have increased the statistical power of our study to uncover the metabolic changes others have seen. However, comparison of multiple datasets from the literature can be misleading due to sampling differences (age, sex, ethnicity, severity and timing of the pathology). Here, our cohort included only Japanese participants. Moreover, our PDR patients are younger and have higher BMI values compared with the control group. Increasing age is a major risk factor for ERM in this adult Japanese population [30]. In this study, we did not adjust for age. BMI is generally higher in individuals with the metabolic syndrome with diabetes mellitus compared with normal control participants, and we were not able to adjust for this variable. In the Japanese blind population, diabetic retinopathy blindness occurred in $15.0 \%$ of males and $10.4 \%$ of females [31]. The unbalanced sex population of PDR patients in the current study may skew results. Another limiting factor of the study is the lack of data regarding the systemic levels of the metabolites analysed. This is particularly relevant with creatine, because systemic levels can be modulated by diet, age and the endogenous production from the liver and kidneys. In this study systemic samples such as blood were not taken routinely for metabolome analysis. A prospective study is needed to address this issue. There is an overlap in vitreous sample metabolite levels between nondiabetic and PDR patients in Fig. 4. In fact, all of the values obtained in control participants are included in the range of 

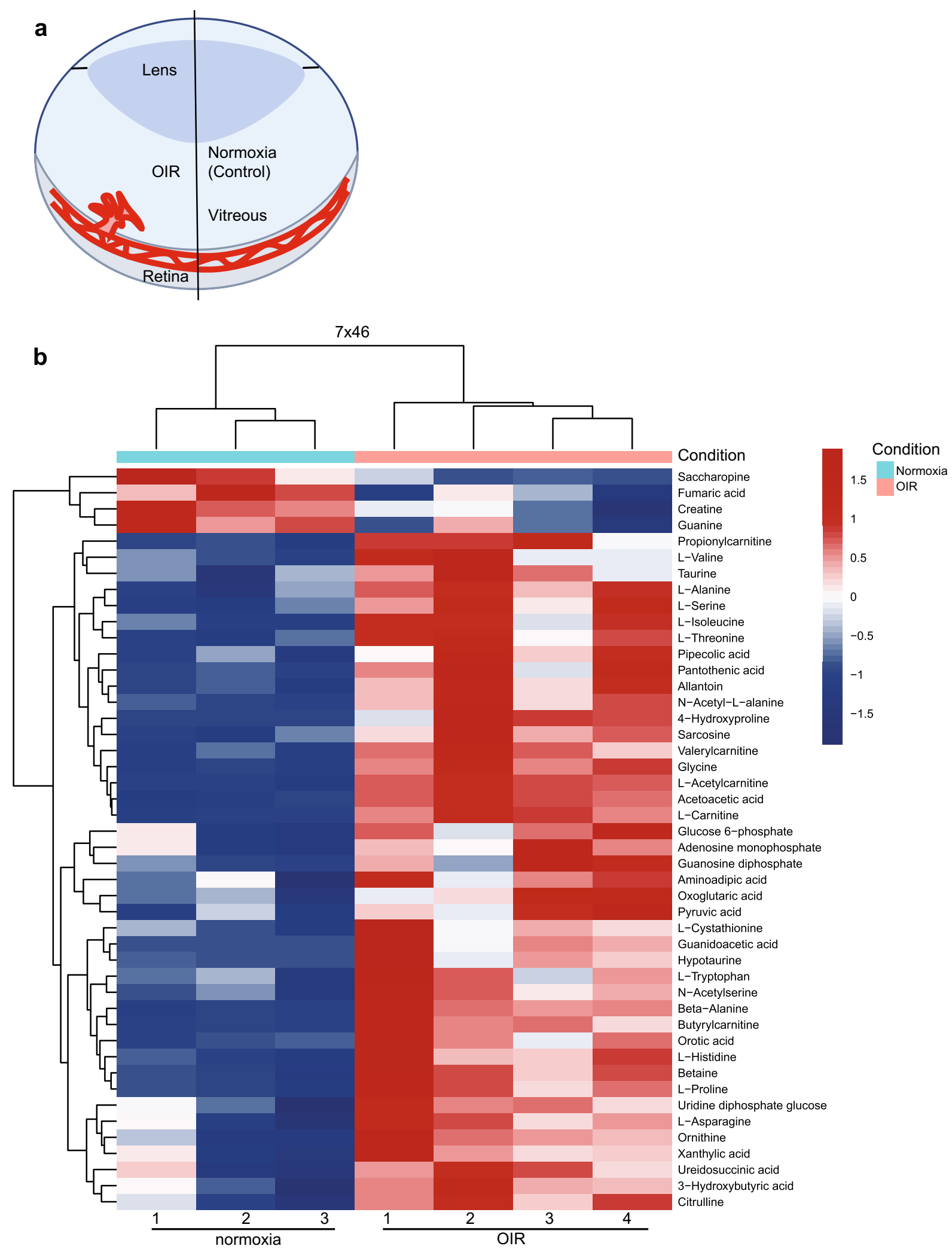

Fig. 5 Unsupervised clustering analysis of samples. (a) Simplified schematic of the eye showing the retinal vasculature in OIR and control normoxic retinas (normoxia). (b) Control normoxic and OIR retinas were well resolved in an unsupervised clustering analysis. Using the significant metabolites $(p<0.05)$ from the comparison, samples were clustered with the complete method and Euclidean distance function 
Fig. 6 The levels of the glycine/ creatine pathway in OIR mouse retina. (a) In OIR mice, creatine levels were decreased in the retina compared with control normoxic retinas ( $n=4,3$, respectively). (b) Glycine levels were increased in the retinas of OIR mice compared with control normoxic retinas $(n=4,3)$. (c, d) Phosphocreatine and creatinine were not significantly different between the two groups $(n=4,3)$. (e)

Schematic of the glycine/creatine pathway. The data were analysed by Student's $t$ test and were expressed as mean $\pm \mathrm{SEM}$. ${ }^{*} p<0.05 ; * * * p<0.001$. FC, fold change vs control

Fig. 7 Creatine supplementation suppressed NV in OIR mice. (ad) Representative retinal whole mounted images after automated deep learning segmentation (yellow, VO; red, NV) from PBSand creatine-treated OIR mice. Scale bar, $1 \mathrm{~mm}$. (e) The NV area in the creatine group was significantly decreased compared with the PBS group $(n=10)$. (f) No significant differences in $\mathrm{VO}$ were detected among the groups $(n=10)$. (g) Body weight

development of both groups from $\mathrm{P} 12$ to $\mathrm{P} 17(n=10)$. The data were analysed by Student's $t$ test and were expressed as mean \pm SEM. $* * p<0.01$. FC, fold change vs control
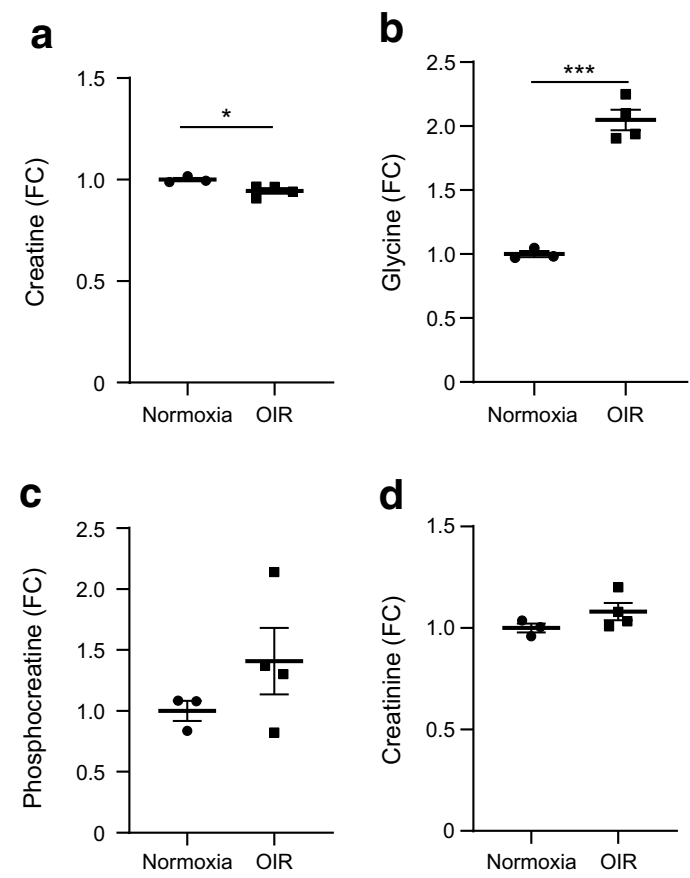

e

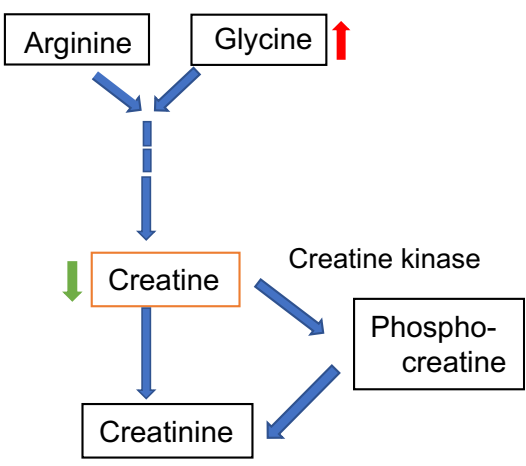

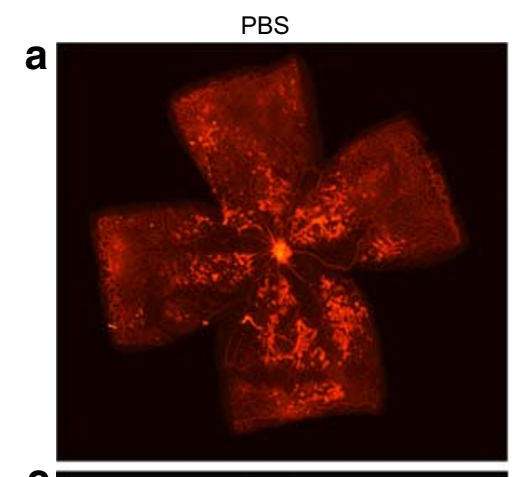
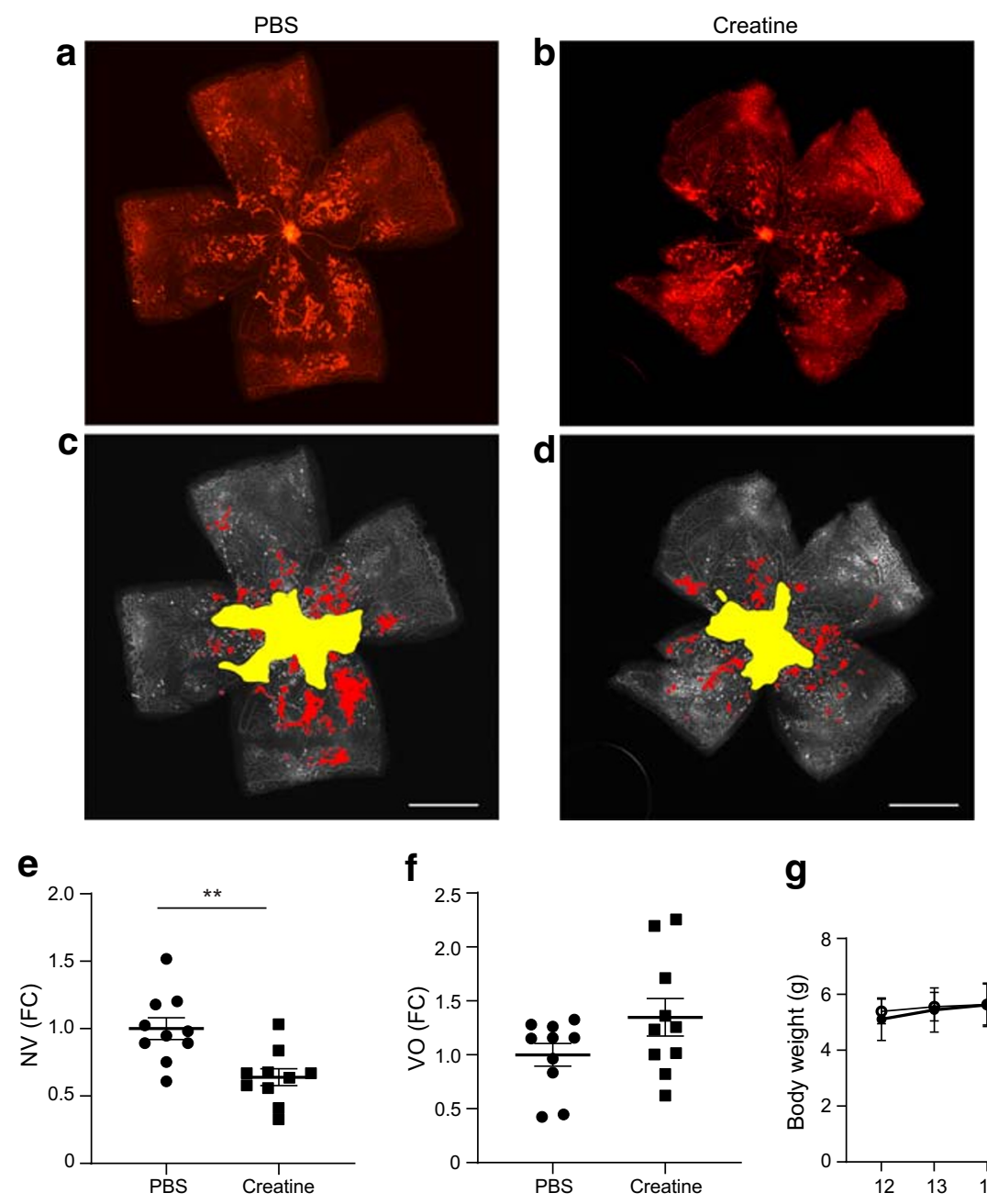

f

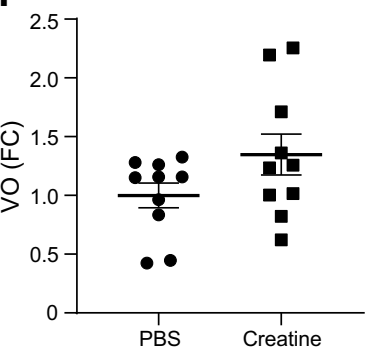

g

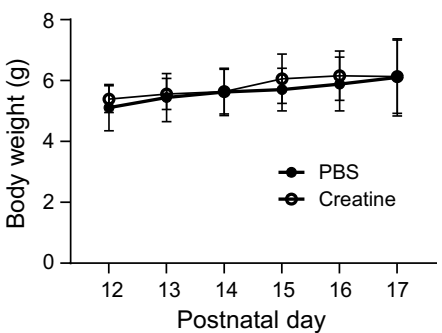


PDR patients. In this regard, it should be noted that both the mean age and the SD are quite different in PDR patients in comparison with non-diabetic control participants.

Another potential concern is that we compared metabolites in human vitreous humour with those in mouse retina, as the volume of mouse vitreous sample was too small to measure metabolites. However, comparisons of vitreous humour vs whole eyes, including retina, have been made in other studies and showed significant correlation [10]. Another limitation is that the OIR model mimics only NV in the retina, but not other aspects of diabetes that precede the NV. However, there is no reliable animal model of PDR.

Creatine as well as pathways involved with creatine were altered in vitreous humour from PDR patients. Creatine was decreased in both human vitreous humour with PDR and OIR retinas. Creatine supplementation decreased NV in OIR. Interestingly, creatine is a by-product of glycine degradation, and glycine and other metabolites of glycine were significantly enriched in PDR vitreous samples. A previous study also showed that in PDR vitreous humour, glycine as well as proline and other amino acids levels were elevated, and they were associated with a decrease in angiogenesis markers in bovine retinal pericytes [29].

PDR patients have elevated intraocular VEGF levels in the aqueous and vitreous fluids [32]. Platelet-derived growth factors (PDGFs) have also been reported to be important proangiogenic factors involved in retinal and corneal NV via activating platelet-derived growth factor receptor (PDGFR) [33, 34]. In addition, a study showed that suppressing PDGFR ameliorated retinal NV in OIR [35]. In our study creatine treatment decreased the expression levels of Vegf- $a$ and Pdgf- $b$ mRNA in the OIR retina (ESM Fig. 4). Thus, suppressing VEGF and PDGF by creatine could contribute to reducing NV. More extensive studies are needed to establish the mechanism of action of creatine in suppressing NV in OIR.

Although creatine supplementation suppressed NV it did not do so by increasing regrowth of physiological vessels in OIR mice. This could be because of a direct effect of creatine inhibition of neo-vessels vs normal vessels, or because creatine improved neuronal metabolism causing reduced signalling for NV. Several studies have reported that the creatine transporter is expressed in endothelial and retinal ganglion cells $[36,37]$. So, differences in expression of these transporters between normal and neovascular endothelial cells could be responsible for different reactions between normal and neovascular vessels. It is also possible that creatine could directly improve retinal neuron metabolism which would decrease signalling for NV. However, this is speculative, and we need further studies to prove these theories.

Creatine has been shown to act as an antioxidant and may reduce inflammation in ageing adults [38]. A study reported that creatine supplementation prevented atrophy of skeletal muscle by attenuating proinflammatory molecule levels [39].
Another study showed that creatine supplementation attenuated TNF- $\alpha$ in the muscle after a $30 \mathrm{~km}$ race in humans [40]. There are many clinical trials examining the effects of creatine supplementation on sarcopenia, osteoporosis, chronic fatigue syndrome, intermittent claudication and depression (https:// clinicaltrials.gov/ct2/home). One study showed that creatine supplementation combined with exercise improved glycaemic control in type 2 diabetic patients [41]. This result suggests that creatine supplementation may be a potential novel preventative treatment for PDR. Although renal adverse effects were not observed in this study, we need further detailed investigations into renal function in any future preclinical or clinical studies of creatine.

In summary, metabolites from the vitreous humour may be useful in detecting potential new pathways and targets for ocular diseases. In this study, we documented that creatine supplementation inhibited pathological NV in a mouse model of retinal NV (OIR), suggesting that creatine may be a potential target in PDR in diabetic patients. Overall, this and other vitreous metabolites datasets may be of value to uncover other PDR pathways.

Acknowledgements Thanks to the NYU Metabolomics Core Resource Laboratory, New York, NY, USA for metabolome analysis for mice.

Data availability The datasets generated and/or analysed during the current study are available from the corresponding author by reasonable request.

Funding The work was supported by grants from the Manpei Suzuki Diabetic Foundation and Alcon Research Institute (YT); the Vision Health Research Network for its contribution to the Montreal SingleCell Academy (GC); Boston Children's Hospital Manton Center for Orphan Disease Research, OFD/BTREC/CTREC Faculty Career Development Grant, and Ophthalmology Foundation, Little Giraffe Foundation, Mass Lions Eye Foundation (ZF); the German Research Foundation (DFG; to BC [CA1940/1-1]); the Wallenberg Clinical Scholars (AH); the Burroughs Wellcome Fund Career Award for Medical Scientists; the Foundation Fighting Blindness; the Canadian Institute of Health Research (CIHR; 390615); the Fonds de Recherche du Québec-Santé (FRQS); the Canadian Child Health Clinician Scientist Program; a CIHR New Investigator Award; the Natural Sciences and Engineering Research Council of Canada (NSERC: RGPIN-201606743) (J-SJ); NIH R24EY024868, EY017017, R01EY01717-13S1 and EY030904-01; the BCH IDDRC (1U54HD090255); Massachusetts Lions Eye Foundation (LEHS); and Grants-in-Aid for Scientific Research (C) 16K11330 and 19K09959 from the Ministry of Education, Culture, Sports, Science and Technology of Japan (YU).

Authors' relationships and activities ST is an employee of Merck Sharp \& Dohme Corp., a subsidiary of Merck \& Co., Kenilworth, NJ, USA, and a stockholder in Merck \& Co., Kenilworth, NJ, USA. The other authors declare that there are no relationships or activities that might bias, or be perceived to bias, their work.

Contribution statement All authors have substantially contributed to conception and design, acquisition of data, or analysis and interpretation of data and drafting the article or revising it critically for important intellectual content. Furthermore, all authors have approved the final version to be published. YU and LEHS are responsible for the integrity of the work as a whole. 


\section{References}

1. Hendrick AM, Gibson MV, Kulshreshtha A (2015) Diabetic retinopathy. Prim Care 42(3):451-464. https://doi.org/10.1016/j.pop. 2015.05.005

2. Fong DS, Ferris FL 3rd, Davis MD, Chew EY (1999) Causes of severe visual loss in the early treatment diabetic retinopathy study: ETDRS report no. 24. Early Treatment Diabetic Retinopathy Study Research Group. Am J Ophthalmol 127(2):137-141. https://doi. org/10.1016/s0002-9394(98)00309-2

3. Porta M, Striglia E (2020) Intravitreal anti-VEGF agents and cardiovascular risk. Intern Emerg Med 15(2):199-210. https://doi. org/10.1007/s11739-019-02253-7

4. Simo R, Hernandez C (2008) Intravitreous anti-VEGF for diabetic retinopathy: hopes and fears for a new therapeutic strategy. Diabetologia 51(9):1574-1580. https://doi.org/10.1007/s00125008-0989-9

5. Liew G, Lei Z, Tan G et al (2017) Metabolomics of diabetic retinopathy. Curr Diab Rep 17(11):102. https://doi.org/10.1007/ s11892-017-0939-3

6. Haines NR, Manoharan N, Olson JL, D'Alessandro A, Reisz JA (2018) Metabolomics analysis of human vitreous in diabetic retinopathy and rhegmatogenous retinal detachment. J Proteome Res 17(7):2421-2427. https://doi.org/10.1021/acs.jproteome.8b00169

7. Lauwen S, de Jong EK, Lefeber DJ, den Hollander A (2017) Omics biomarkers in ophthalmology. Invest Ophthalmol Vis Sci 58(6): BIO88-BIO98. https://doi.org/10.1167/iovs.17-21809

8. Yoshimura T, Sonoda KH, Sugahara M et al (2009) Comprehensive analysis of inflammatory immune mediators in vitreoretinal diseases. PLoS One 4(12):e8158. https://doi.org/10. 1371/journal.pone.0008158

9. Barba I, Garcia-Ramirez M, Hernandez C et al (2010) Metabolic fingerprints of proliferative diabetic retinopathy: an ${ }^{1} \mathrm{H}-\mathrm{NMR}$-based metabonomic approach using vitreous humor. Invest Ophthalmol Vis Sci 51(9):4416-4421. https://doi.org/10.1167/iovs.10-5348

10. Paris LP, Johnson CH, Aguilar E et al (2016) Global metabolomics reveals metabolic dysregulation in ischemic retinopathy. Metabolomics 12:15. https://doi.org/10.1007/s11306-015-0877-5

11. Bailey JD, Diotallevi M, Nicol T et al (2019) Nitric oxide modulates metabolic remodeling in inflammatory macrophages through TCA cycle regulation and itaconate accumulation. Cell Rep 28(1): 218-230.e7. https://doi.org/10.1016/j.celrep.2019.06.018

12. Serrano N, Ling Y, Bahieldin A, Mahfouz MM (2019) Thermopriming reprograms metabolic homeostasis to confer heat tolerance. Sci Rep 9(1):181. https://doi.org/10.1038/s41598-01836484-z

13. Chong J, Wishart DS, Xia J (2019) Using MetaboAnalyst 4.0 for comprehensive and integrative metabolomics data analysis. Curr Protoc Bioinformatics 68(1):e86. https://doi.org/10.1002/cpbi.86

14. Pang Z, Chong J, Li S, Xia J (2020) MetaboAnalystR 3.0: toward an optimized workflow for global metabolomics. Metabolites 10(5). https://doi.org/10.3390/metabo10050186

15. Subramanian A, Tamayo P, Mootha VK et al (2005) Gene set enrichment analysis: a knowledge-based approach for interpreting genome-wide expression profiles. Proc Natl Acad Sci U S A 102(43):15545-15550. https://doi.org/10.1073/pnas.0506580102

16. Smith LE, Wesolowski E, McLellan A et al (1994) Oxygeninduced retinopathy in the mouse. Invest Ophthalmol Vis Sci 35(1):101-111

17. Stahl A, Connor KM, Sapieha P et al (2009) Computer-aided quantification of retinal neovascularization. Angiogenesis 12(3):297301. https://doi.org/10.1007/s10456-009-9155-3

18. Connor KM, Krah NM, Dennison RJ et al (2009) Quantification of oxygen-induced retinopathy in the mouse: a model of vessel loss, vessel regrowth and pathological angiogenesis. Nat Protoc 4(11): 1565-1573. https://doi.org/10.1038/nprot.2009.187
19. Pacold ME, Brimacombe KR, Chan SH et al (2016) A PHGDH inhibitor reveals coordination of serine synthesis and one-carbon unit fate. Nat Chem Biol 12(6):452-458. https://doi.org/10.1038/ nchembio. 2070

20. Chen WW, Freinkman E, Wang T, Birsoy K, Sabatini DM (2016) Absolute quantification of matrix metabolites reveals the dynamics of mitochondrial metabolism. Cell 166(5):1324-1337.e11. https:// doi.org/10.1016/j.cell.2016.07.040

21. Simon-Manso Y, Lowenthal MS, Kilpatrick LE et al (2013) Metabolite profiling of a NIST Standard Reference Material for human plasma (SRM 1950): GC-MS, LC-MS, NMR, and clinical laboratory analyses, libraries, and web-based resources. Anal Chem 85(24):11725-11731. https://doi.org/10.1021/ac402503m

22. Smith CA, O'Maille G, Want EJ et al (2005) METLIN: a metabolite mass spectral database. Ther Drug Monit 27(6):747-751. https://doi.org/10.1097/01.ftd.0000179845.53213.39

23. Chantranupong L, Saulnier JL, Wang W, Jones DR, Pacold ME, Sabatini BL (2020) Rapid purification and metabolomic profiling of synaptic vesicles from mammalian brain. eLife 9:e59699. https:// doi.org/10.7554/eLife.59699

24. Gong Y, Shao Z, Fu Z et al (2016) Fenofibrate inhibits cytochrome P450 epoxygenase $2 \mathrm{C}$ activity to suppress pathological ocular angiogenesis. EBioMedicine 13:201-211. https://doi.org/10.1016/ j.ebiom.2016.09.025

25. Stahl A, Connor KM, Sapieha P et al (2010) The mouse retina as an angiogenesis model. Invest Ophthalmol Vis Sci 51(6):2813-2826. https://doi.org/10.1167/iovs.10-5176

26. Xiao S, Bucher F, Wu Y et al (2017) Fully automated, deep learning segmentation of oxygen-induced retinopathy images. JCI Insight 2(24):e97585. https://doi.org/10.1172/jci.insight.97585

27. Tomita Y, Cakir B, Liu CH et al (2020) Free fatty acid receptor 4 activation protects against choroidal neovascularization in mice. Angiogenesis 23(3):385-394. https://doi.org/10.1007/s10456-02009717-x

28. Stahl A, Chen J, Sapieha P et al (2010) Postnatal weight gain modifies severity and functional outcome of oxygen-induced proliferative retinopathy. Am J Pathol 177(6):2715-2723. https:// doi.org/10.2353/ajpath.2010.100526

29. Vidhya S, Ramya R, Coral K, Sulochana KN, Bharathidevi SR (2018) Free amino acids hydroxyproline, lysine, and glycine promote differentiation of retinal pericytes to adipocytes: a protective role against proliferative diabetic retinopathy. Exp Eye Res 173:179-187. https://doi.org/10.1016/j.exer.2018.05.004

30. Kawasaki R, Wang JJ, Sato H et al (2009) Prevalence and associations of epiretinal membranes in an adult Japanese population: the Funagata study. Eye (Lond) 23(5):1045-1051. https://doi.org/10. 1038/eye.2008.238

31. Morizane Y, Morimoto N, Fujiwara A et al (2019) Incidence and causes of visual impairment in Japan: the first nation-wide complete enumeration survey of newly certified visually impaired individuals. Jpn J Ophthalmol 63(1):26-33. https://doi.org/10.1007/ s10384-018-0623-4

32. Wirostko B, Wong TY, Simo R (2008) Vascular endothelial growth factor and diabetic complications. Prog Retin Eye Res 27(6):608621. https://doi.org/10.1016/j.preteyeres.2008.09.002

33. Hou X, Kumar A, Lee C et al (2010) PDGF-CC blockade inhibits pathological angiogenesis by acting on multiple cellular and molecular targets. Proc Natl Acad Sci U S A 107(27):12216-12221. https://doi.org/10.1073/pnas.1004143107

34. Cao R, Brakenhielm E, Li X et al (2002) Angiogenesis stimulated by PDGF-CC, a novel member in the PDGF family, involves activation of PDGFR- $\alpha \alpha$ and $-\alpha \beta$ receptors. FASEB J 16(12):15751583. https://doi.org/10.1096/fj.02-0319com

35. Zhou L, Sun $X$, Huang $Z$ et al (2018) Imatinib ameliorated retinal neovascularization by suppressing PDGFR- $\alpha$ and PDGFR- $\beta$. Cell 
Physiol Biochem 48(1):263-273. https://doi.org/10.1159/ 000491726

36. de Souza CF, Kalloniatis M, Christie DL, Polkinghorne PJ, McGhee CN, Acosta ML (2012) Creatine transporter immunolocalization in aged human and detached retinas. Invest Ophthalmol Vis Sci 53(4):1936-1945. https://doi.org/10.1167/iovs.11-8462

37. Tachikawa M, Hosoya K, Ohtsuki S, Terasaki T (2007) A novel relationship between creatine transport at the blood-brain and blood-retinal barriers, creatine biosynthesis, and its use for brain and retinal energy homeostasis. Subcell Biochem 46:83-98. https://doi.org/10.1007/978-1-4020-6486-9 5

38. Candow DG, Forbes SC, Chilibeck PD, Cornish SM, Antonio J, Kreider RB (2019) Effectiveness of creatine supplementation on aging muscle and bone: focus on falls prevention and inflammation. J Clin Med 8(4):488. https://doi.org/10.3390/jcm8040488

39. Cella PS, Marinello PC, Borges FH et al (2019) Creatine supplementation in Walker-256 tumor-bearing rats prevents skeletal muscle atrophy by attenuating systemic inflammation and protein degradation signaling. Eur J Nutr 59(2):661-669. https://doi.org/ 10.1007/s00394-019-01933-6

40. Santos RV, Bassit RA, Caperuto EC, Costa Rosa LF (2004) The effect of creatine supplementation upon inflammatory and muscle soreness markers after a 30km race. Life Sci 75(16):1917-1924. https://doi.org/10.1016/j.lfs.2003.11.036

41. Gualano B, DESP V, Roschel $\mathrm{H}$ et al (2011) Creatine in type 2 diabetes: a randomized, double-blind, placebo-controlled trial. Med Sci Sports Exerc 43(5):770-778. https://doi.org/10.1249/MSS. 0b013e3181fcee7d

Publisher's note Springer Nature remains neutral with regard to jurisdictional claims in published maps and institutional affiliations. 